\title{
Automated Analysis of Hop Bittering Components with High Performance Liquid Chromatography Using Precolumn Switching
}

\author{
Miyoko ONo*, Youichi KaKUdo* and Suehiro UEDA** \\ * Research Institute of Alcoholic Beverages and Fermentation Technology, Suntory Ltd., \\ Shimamoto-cho, Mishima-gun, Osaka 618 \\ **Analytical Applications Department, Shimadzu Corporation, \\ Nishinokyo-Kuwabara-cho, Nakagyo-ku, Kyoto 604
}

\begin{abstract}
An automated method based on on-line sample enrichment by precolumn switching has been developed for the determination of hop bittering components in beer and wort. A combination of two columns was used: a tetramethylolmethanetriacrylate type for trapping and an ODS silica type for final separation. Iso- $\alpha$-acids and $\alpha$ acids were determined with similar results by the conventional method, based on off-line sample enrichment (liquidliquid extraction and concentration). The analysis was carried out with good reproducibilities (rsd less than $3 \%$ ). Precolumn switching is distinguished by injection of beer and wort samples without preteatment, and is eminently suited for automated routine analysis in breweries.
\end{abstract}

Keywords Automated HPLC, beer, wort, precolumn switching, direct injection, on-line sample enrichment

Hops, used in a brewery are the dried blossoms of the female hop plant(humulus lupulus). The bitter acids of hops mainly consists of $\alpha$-acids(I) and $\beta$ acids(II) (see Fig. 1). The $\alpha$-acids make the largest contribution by their chemical transformation during the brewing process to iso- $\alpha$-acids(III), which impart most of the refreshing, clean bitterness to beer. Each of the important hop bittering components, $\alpha$-, $\beta$ - and iso- $\alpha$-acids, has three major analogs (see Fig. 1).<smiles>[R]C(=O)C1=C(O)C(CC=C(C)C)=C(O)C(O)(CC=C(C)C)C1=O</smiles><smiles>[R]C(=O)C1=C(O)C(CC=C(C)C)=C(O)C(C=CC(C)C)(C=CC(C)C)C1=O</smiles>

(III)<smiles>[R]C(=O)C1=C(C)C(O)(C(=O)/C=C/C(C)C)C(CC=C(C)C)C1=O</smiles>

III

$\begin{array}{llll} & \alpha \text {-acids } & \beta \text {-acids } & \text { iso- } \alpha \text {-acids } \\ \mathrm{R}=\mathrm{CH}\left(\mathrm{CH}_{3}\right)_{2} & \text { cohumulone } & \text { colupulone } & \text { isocohumulone } \\ \mathrm{R}=\mathrm{CH}_{2} \mathrm{CH}\left(\mathrm{CH}_{3}\right)_{2} & \text { humulone } & \text { lupulone } & \text { isohumulone } \\ \mathrm{R}=\mathrm{CH}\left(\mathrm{CH}_{3}\right) \mathrm{CH}_{2} \mathrm{CH}_{3} & \text { adhumulone } & \text { adlupulone } & \text { isoadhumulone }\end{array}$

Fig. 1 Structures of hop bittering components.
Analysis of these compounds as individuals or as a group is important for a brewery for both commercial appeal and quality control in the brewing process. We have developed a simultaneous analysis for $\alpha-, \beta$ - and iso- $\alpha$-acids by HPLC. ${ }^{1,2}$ The technique is applicable not only to hops and hop products but also wort and beer in all stages of the brewing process. However, the complexity of the sample and the low concentration made sample enrichment indispensable before the HPLC analysis. We investigated the application of sample enrichment and clean-up method in relation to preparation, reproducibility, automatization and time requirement, comparing on-line with off-line sample enrichment methods. On-line sample enrichment in HPLC analysis of beer and wort samples could be achieved by precolumn switching techniques.

\section{Experimental}

\section{Reagents}

$\beta$-Phenylchalkone (internal standard [IS]) and triethylamine were obtained from Wako Pure Chemicals (Tokyo). Cohumulone, humulonetadhumulone, colupulone and lupulonetadluplone were fractionated with silica gel chromatography from $\mathrm{CO}_{2}$ hop extracts and purified with reserved-phase ODS column chromatography. Their purity was checked by our HPLC method, melting point, IR and mass spectrometry. ${ }^{1,2}$ 


\section{Equipment}

All HPLC equipment used is from the Shimadzu Co., Ltd.; it comprised three model LC-6A with a SIL-6A automatic sample injector and a CTO-6A column thermostatting oven. Detection was monitored by an SPD-6A spectrophotometric detector and a CR3A data processor with chromatogram memory and auto-zero adjustments. The system was additionally equipped with a FCV-2AH flow change valve for column switching. Overall control of the system was via the model SCL-6A system controller.

\section{Column}

The analytical column was a Shim-pack CLCODS/H (4.6 mm I.D. $\times 250 \mathrm{~mm}$ L., $5 \mu \mathrm{m}$, Shimadzu). The quality of the Shim-pack CLC-ODS/H is carefully controlled so that each analog of iso- $\alpha$-acids can be separated. The precolumn for the sample clean-up and concentration was the Shim-pack SPCRP1 (4.0 mm I.D. $\times 30 \mathrm{~mm} \mathrm{L.,} \mathrm{Shimadzu).}$

\section{Off-line sample preparation by liquid-liquid extraction}

Liquid-liquid extraction was performed according to the ASBC method. ${ }^{3}$ A wort sample or a degassed beer sample $(10.0 \mathrm{ml})$ was transferred into a $100 \mathrm{ml}$ separatory funnel, acidified with $0.5 \mathrm{ml}$ of $6 \mathrm{M} \mathrm{HCl}$, and extracted with $20.0 \mathrm{ml}$ of isooctane by mechanical shaking for $30 \mathrm{~min}$ and standing for $30 \mathrm{~min}$. Twelve $\mathrm{ml}$ of the isooctane layer was completely evaporated under reduced pressure. The residue was dissolved in $0.5 \mathrm{ml}$ of methanol containing the internal standard, $\beta$ phenylchalkone at $6.25 \mathrm{mg} / 100 \mathrm{ml}$ and $10 \mu \mathrm{l}$ of the prepared sample were injected onto the analytical column.

\section{On-line sample preparation by precolumn switching}

A wort sample or a degassed beer sample $(1.0 \mathrm{ml})$ was mixed with $0.2 \mathrm{ml}$ of methanol containing the internal standard at $7.5 \mathrm{mg} / 100 \mathrm{ml}$ and $120 \mu \mathrm{l}$ of the sample were injected onto the precolumn.

\section{Mobile phase}

The analysis was carried out by three mobile phases for the analysis (solvent A, B and C) were prepared according to the previous report. ${ }^{2}$ Solvent $A$ was methanol and water (77.5:22.5) containing $85 \%$ phosphoric acid $(1.7 \mathrm{~g} / 100 \mathrm{ml})$ and $10 \%$ tetraethylammonium hydroxide $(2.95 \mathrm{~g} / 100 \mathrm{ml})$. Solvent B was methanol. Solvent $\mathrm{C}$ was methanol and water (77.5:22.5) containing $85 \%$ phosphoric acid $(0.5 \mathrm{~g} / 100$ $\mathrm{ml})$ and $10 \%$ tetraethylammonium hydroxide $(2.95 \mathrm{~g} /$ $100 \mathrm{ml}$ ). Apparent $\mathrm{pH}$ of solvent $\mathrm{C}$ was 4.85 . Mobile phases for sample enrichment were methanol and $0.1 \%$ phosphoric acid $(20: 80, \mathrm{v} / \mathrm{v})($ solvent $\mathrm{D}$ for purge phase) and $50 \%$ aq. methanol/triethylamine (100:1) (solvent $\mathrm{E}$ for washing).

\section{Precohumn switching technique}

The schematic set-up of precolumn switching is shown in Fig. 2. Sample volumes of 120 or $180 \mu \mathrm{l}$ were loaded onto the sample loop of the automatic sample injector and then carried to the precolumn, which had been conditioned with the purge phase (solvent $D$ )

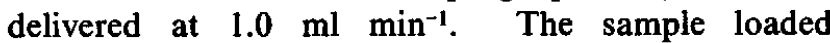
precolumn was washed for a further 5 min with the purge phase to eliminate accompanying water-soluble co-products. After switching of the precolumn into the flow line of the chromatographic system, the enriched sample was eluted onto the analytical column with the mobile phase for analysis delivered at $1.5 \mathrm{ml} \mathrm{min}$. The time program of precolumn switching is shown in Fig. 3. During the analysis, the precolumn was washed with solvent $E$ and then reconditioned with solvent $D$ for the next analysis.

\section{Chromatography}

Separation was carried out by gradient elution. The slightly modified time program of the HPLC analysis

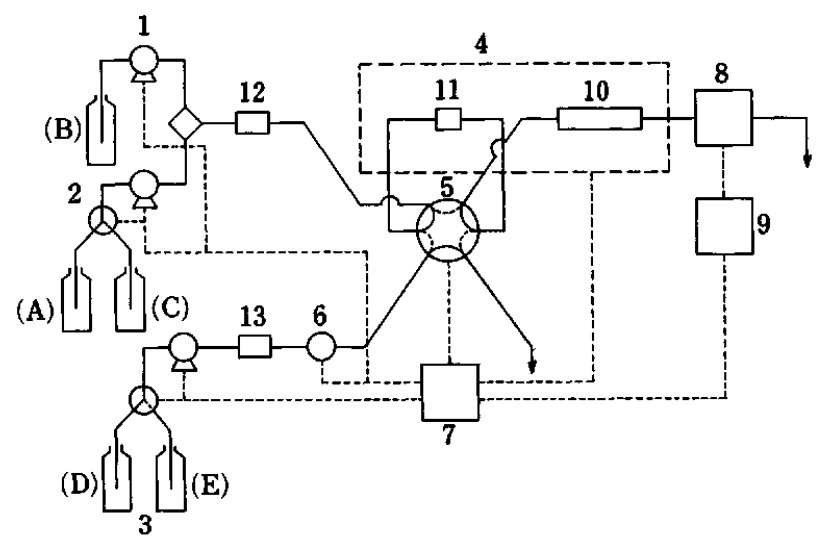

Fig. 2 Flow diagram of the fully automated HPLC for hop bittering components. 1, pump; 2, mobile phase for analysis; 3, mobile phase for preparation; 4, column oven; 5 , flow changeover valve; 6 , automatic sample injector; 7 , system controller; 8, spectrophotometric detector; 9, data processor; 10, analytical column; 11, precolumn for sample preparation; 12, precolumn for protecting analytical column (Shimadzu ODS guard column); 13, precolumn for trapping impurities in mobile phase (SPC-RP1).

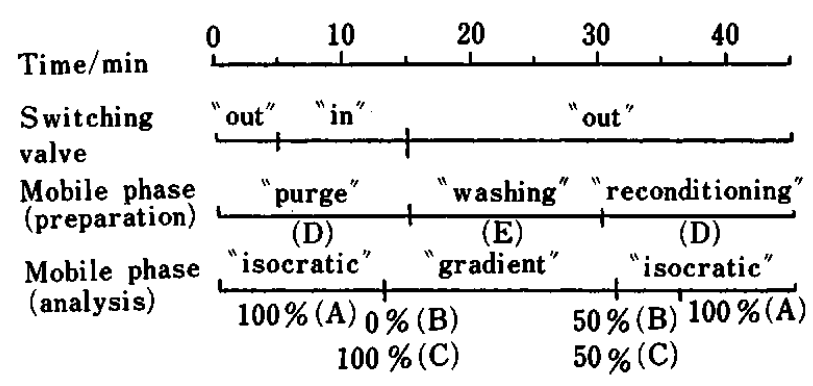

Fig. 3 Time program of the fully automated HPLC for hop bitterring components. 
described in the previous report was used (see Fig. 3). The detection wavelength was changed from $270 \mathrm{~nm}$ to $302 \mathrm{~nm}$ at $19 \mathrm{~min}$ after injection.

\section{Calibration and calculation}

Calibration and calculation for $\alpha$-acids and $\beta$-acid were carried out using authentic standard solution. Those for iso- $\alpha$-acids were performed according to the ASBC method BEER-23, B. ${ }^{4}$

\section{Results and Discussion}

\section{On-line sample enrichment using precolumn switching}

The HPLC method previously described ${ }^{1,2}$ can simultaneously separate and quantify all analogs of $i$ so- $\alpha$-acids, $\alpha$-acids and $\beta$-acids, the nine main hop bittering components, in less than 30 minutes. In view of the different lipophilic properties of these substances, their low concentration in the sample and the complex matrices of the sample, sample clean-up and enrichment was indispensable before the HPLC analysis. Consequently, precolumn switching technique was applied to replace these sample clean-up and enrichment techniques of time-consuming extraction, evaporation and redissolution steps. The effect of the $\mathrm{pH}$ and the concentration of methanol in the purge phase on the on-line enrichment of iso- $\alpha$ acids in beer was investigated, because it was suggested that the enrichment for them was most difficult due to their $\mathrm{p} K_{\mathrm{a}}$ values being lower than those of $\alpha$-acids and $\beta$-acids.

Figure 4 shows the effect of the $\mathrm{pH}$ of phosphoric acid aqueous solution in the purge phase on the enrichment of the iso- $\alpha$-acids in beer. The concentration of methanol was fixed at $20 \%$ in this investigation.

Figure 5 shows the effect of the concentration of methanol mixed with phosphoric acid aqueous solution (pH 2.5) for the purge phase on the enrichment.

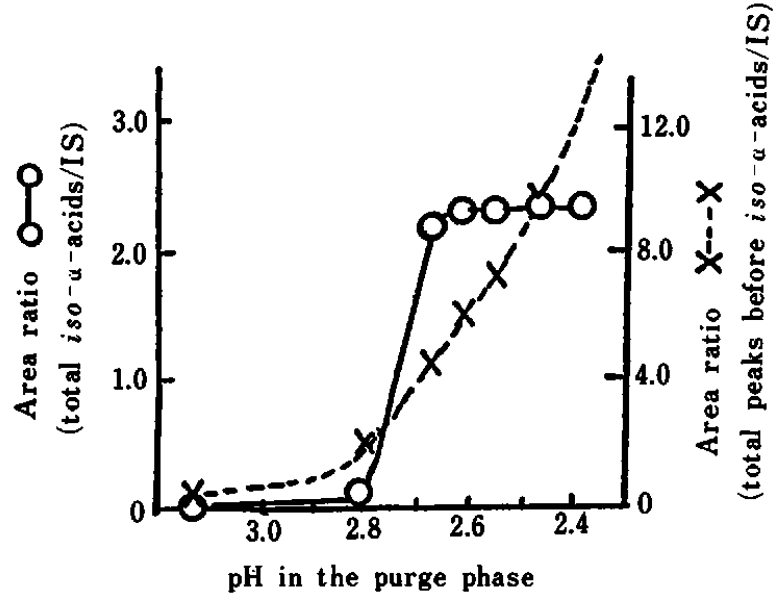

Fig. 4 Effect of the $\mathrm{pH}$ in the purge phase on the enrichment.

These results show that the lower the $\mathrm{pH}$ and the concentration of methanol, the more the enrichment of iso- $\alpha$-acids. However, these phenomena were accompanied with the further enrichment of components which were eluted before iso- $\alpha$-acids. From these results, we selected the purge phase of methanol $/ 0.1 \%$ phosphoric acid (20:80, pH 2.5-2.6).

Figures 6 and 7 show the comparison of HPLC analysis between the on-line sample enrichment (precolumn switching) and the off-line enrichment (liquid-liquid extraction and concentration).

As is shown in Table 1, the same results were obtained by the on-line sample enrichment using precolumn switching and by the off-line sample enrichment performed in the previously-reported method. ${ }^{2}$

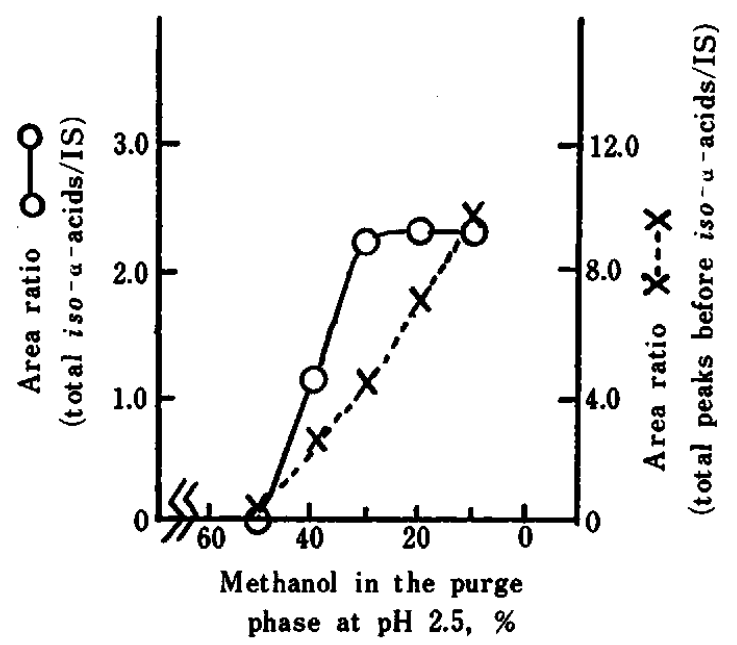

Fig. 5 Effect of the concentration of methanol in the purge phase at pH 2.5 on the enrichment.

(A)

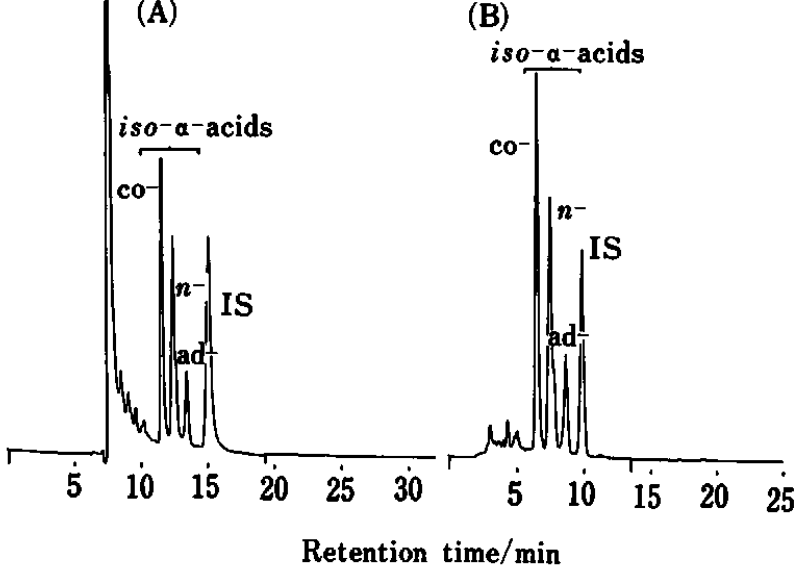

Fig. 6 Comparison of the HPLC analysis of on-line sample enrichment with off-line sample enrichment for beer: $A$, on-line sample enrichment; B, off-line sample enrichment. The two peaks for $n$-analog of iso- $\alpha$-acid are the cis and trans forms. 


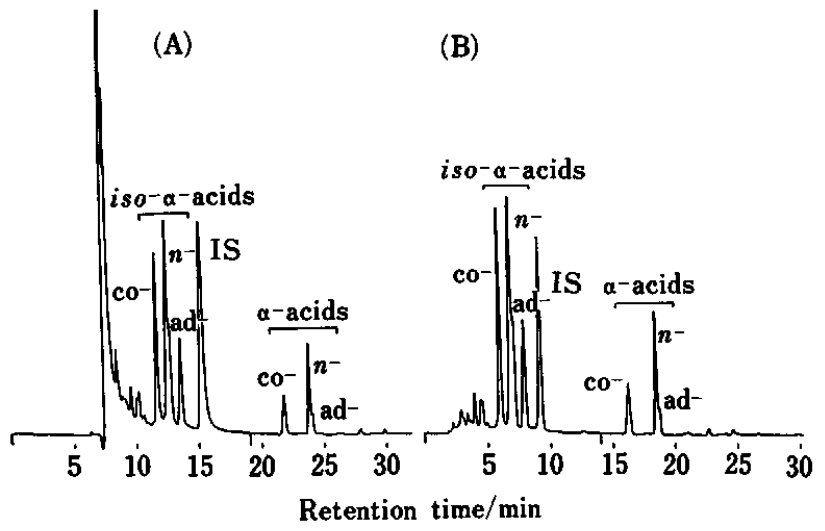

Fig. 7 Comparison of the HPLC analysis of on-line sample enrichment with off-line sample enrichment for wort: A, on-line sample enrichment; $B$, off-line sample enrichment. The two peaks for $n$-analog of $i s o-\alpha$-acids are the cis and trans forms.

In case of wort sample, small peaks eluted after the $\alpha$-acids were observed. They corresponded to the small amount of each analog of $\beta$-acids. So, $\beta$-acids presented in a sample can be simultaneously determined, if they are over $0.1 \mathrm{ppm}$.

Precision of the automated HPLC analysis using precolumn switching

Precolumn switching was distinguished by a high level of precision (Table 2). The relative standard deviations of 6 repeated injections of $120 \mu \mathrm{l}$ of three beers of different types ranged between $1 \%$ and $3 \%$ in cases of each component of iso- $\alpha$-acid, and total iso- $\alpha$ acids. The same results were obtained in cases of iso- $\alpha$ acids and $\alpha$-acids in wort.

We have developed the fully automated HPLC method for the main hop bittering components in beer and wort using precolumn switching. This method can overcome sample clean-up and enrichment techniques usually encountered with time-consuming and errorprone extraction, evaporation and redissolution steps which normally were performed before the HPLC analysis. One cycle of the analysis time requires less than $\mathbf{4 0}$ min to determine the main nine hop bittering components, namely, all analogs of iso- $\alpha$-acids, $\alpha$-acids and $\beta$-acids. The precision of this technique is excellent and is comparable to that of the previouslyreported method. The lifetime of the precolumn is sufficiently long that, after analyses of over 500 samples, the sample preparation and column efficiency are not reduced. The automated HPLC method is sufficiently simple, rapid and reproducible for routine analyses in the brewery.
Table 1 Comparison of the concentrations of hop bittering components by on-line sample enrichment and by off-line enrichment

\begin{tabular}{lccrrr}
\hline & \multicolumn{4}{c}{ Contents, ppm } \\
& \multicolumn{3}{c}{ Beer } & \multicolumn{2}{c}{ Wort } \\
\cline { 2 - 3 } \cline { 5 - 6 } & Off & On & & Off & On \\
\hline Isocohumolone & 9.8 & 9.8 & 6.2 & 6.1 \\
Isohumulone & 9.3 & 9.9 & & 10.1 & 11.0 \\
Isoadhumulone & 3.7 & 3.3 & 4.5 & 3.7 \\
Total $i s o-\alpha$-acids & 22.8 & 23.0 & 20.8 & 20.8 \\
Cohumulone & & & & 3.5 & 3.5 \\
Humulone & & & & 6.2 & 6.2 \\
Adhumulone & & & & 1.0 & 1.0 \\
Total $\alpha$-acids & & & 10.7 & 10.7 \\
\hline
\end{tabular}

Table 2 Precision of the fully automated HPLC analysis for beer

\begin{tabular}{|c|c|c|c|c|c|}
\hline \multicolumn{2}{|c|}{ Beer A } & \multicolumn{2}{|c|}{$\begin{array}{c}\text { Contents, ppm } \\
\text { Beer B } \\
\end{array}$} & \multicolumn{2}{|c|}{ Beer C } \\
\hline Mean & rsd, $\%$ & Mean & rsd, $\%$ & Mean & rsd, $\%$ \\
\hline \multicolumn{6}{|c|}{ Isocohumulone } \\
\hline 6.5 & 1.9 & 10.1 & 1.0 & 17.0 & 3.1 \\
\hline \multicolumn{6}{|c|}{ Isohumulone } \\
\hline 8.4 & 2.0 & 9.6 & 1.3 & 16.5 & 3.2 \\
\hline \multicolumn{6}{|c|}{ Isoadhumulone } \\
\hline 2.5 & 1.6 & 3.8 & 1.2 & 6.2 & 2.9 \\
\hline \multicolumn{6}{|c|}{ Total iso- $\alpha$-acids } \\
\hline 17.4 & 1.9 & 23.5 & 1.2 & 39.7 & 3.0 \\
\hline
\end{tabular}

rsd, relative standard deviation.

\section{References}

1. M. Ono, Y. Kakudo, Y. Yamamoto, K. Nagami and J. Kumada, J. Am. Soc. Brew. Chem., 42, 167 (1984).

2. M. Ono, Y. Kakudo, Y. Yamamoto, K. Nagami and J. Kumada, J. Am. Soc. Brew. Chem., 43, 136 (1985).

3. American Society of Brewing Chemists, "Methods of Analysis, 7th ed. Beer-23, A, The Society, St. Paul, (1976).

4. American Society of Brewing Chemists, "Method of Analysis", 7th ed. Beer-23, B, The Society, St. Paul, (1976).

(Received January 22, 1986)

(Accepted April 30, 1986) 